\title{
Arterio-ureteral fistula: a nationwide cross-sectional questionnaire analysis
}

\author{
Tycho M. T. W. Lock ${ }^{1}$ (D) Kyara Kamphorst ${ }^{1} \cdot$ Roderick C. N. van den Bergh $^{2} \cdot$ Frans L. Moll $^{3}$. Jean-Paul P. M. de Vries ${ }^{4}$. \\ Rob T. H. Lo . Gérard A. P. de Kort $^{5} \cdot$ Rutger C. G. Bruijnen ${ }^{5} \cdot$ Pieter Dik $^{6,7} \cdot$ Simon Horenblas ${ }^{8} \cdot$ Laetitia M. O. de Kort $^{1}$
}

Received: 4 November 2021 / Accepted: 10 December 2021 / Published online: 22 January 2022

(c) The Author(s) 2022

\begin{abstract}
Purpose Arterio-ureteral fistula (AUF) is an uncommon diagnosis, but potentially lethal. Although the number of reports has increased over the past two decades, the true incidence and contemporary urologists' experience and approach in clinical practice remains unknown. This research is conducted to provide insight in the incidence of AUF in The Netherlands, and the applied diagnostic tests and therapeutic approaches in modern practice.

Methods A nationwide cross-sectional questionnaire analysis was performed by sending a survey to all registered Dutch urologists. Data collection included information on experience with patients with AUF; and their medical history, diagnostics, treatment, and follow-up, and were captured in a standardized template by two independent reviewers. Descriptive statistics were used.

Results Response rate was $62 \%$ and 56 AUFs in 53 patients were reported between 2003 and 2018. The estimated incidence of AUF in The Netherlands in this time period is 3.5 AUFs per year. Hematuria was observed in all patients; $9 \%$ intermittent microhematuria, and $91 \%$ presenting with, or building up to massive hematuria. For the final diagnosis, angiography was the most efficient modality, confirming diagnosis in 58\%. Treatment comprised predominantly endovascular intervention. Conclusion The diagnosis AUF should be considered in patients with persistent intermittent or massive hematuria.
\end{abstract}

Keywords Hematuria $\cdot$ Stents $\cdot$ Arterio-ureteral fistula $\cdot$ Endovascular procedures $\cdot$ Incidence

\section{Abbreviations \\ ACF Arterio-conduit fistula \\ AUF Arterio-ureteral fistula \\ CT Computed tomography}

\section{Introduction}

Arterio-ureteral fistula (AUF) is a rare but potentially lethal complication, where a direct connection between artery or vascular graft and ureter exists. First clinical presentation is often intermittent hematuria, without any additional symptoms, which frequently ceases without any treatment $[1,2]$. AUF may also present with massive hematuria, especially during endoscopic ureteral instrumentation and/or stent replacement.

Tycho M. T. W. Lock and Kyara Kamphorst contributed equally to this paper.

Tycho M. T. W. Lock

M.T.W.T.Lock@umcutrecht.nl

Extended author information available on the last page of the article
Different risk factors for AUF have previously been described [1]: a combination of arterial and ureteral stenting, arterial pseudo-aneurysm, previous urinoma formation, previous radiation, chronically infected tissue, and abdominal (vascular) surgery. This may lead to friction due to arterial pulsations against a ureter with an indwelling stent of frail and less flexible tissue.

Neither the European Association of Urology (EAU) nor the American Urological Association (AUA) include recommendations on AUF diagnosis or treatment.

We observed an increase in the incidence of AUF in our personal practice, which was confirmed by the reports in medical literature.

The aim of the current study is to estimate true incidence in The Netherlands, contemporarily applied diagnostic, and therapeutical approaches.

A cross-sectional questionnaire approach was applied, in which a questionnaire was sent to all registered Dutch urologists. We focused on the incidence in The Netherlands, and the contemporary diagnostic modalities and treatment options. Based on the results of the questionnaire and in the absence of 
guidelines, recommendations and algorithm for clinical urologic practice will be presented.

\section{Materials and methods}

\section{Patients}

A digital questionnaire was sent to all $(n=398)$ registered Dutch urologists in December 2018 via the Dutch Urology Association (NVU). A reminder was sent out in February 2019. Questions included years in practice, and the encounter with patients suggestive of AUF in the period 2003-2018 (Supplementary Fig. 1).

Anonymized details of patients were retrospectively collected via a standard item list (Supplementary Fig. 2) by two independent reviewers (TL and KK). Details included patient details, and data on diagnostics and treatment.

\section{Definitions}

AUF was defined as the confirmed presence of an abnormal passageway between a ureter and any artery or a previously inserted vascular graft. Only cases of confirmed cases of AUF were included. Excluded from analysis were cases describing fistulas between the ureter and any venous structure, fistulas between the bladder and an artery, cases in which ureteral damage was observed but no true fistula, and fistulas between the intestinal part of an ileal conduit urinary diversion and an artery.

End points were death or loss to follow-up. Death was defined as AUF specific (due to massive hemorrhage or infection directly post-diagnosis) or death due to other causes.

\section{Ethics}

The Ethics Committee of the University Medical Center Utrecht approved this study (reference number WAG/ mb/19/042283). The Dutch Urological Association approved questionnaire distribution among urologists. The need for individual consent was waived.

\section{Statistical analysis}

Descriptive statistics were used (SPSS version 27.0).

\section{Results}

\section{Questionnaire}

A total of 247 urologists of the 398 registered urologists in The Netherlands completed the questionnaire, amounting to a response rate of $62 \%$. The years of practice in this group ranged from 1 to 30 years, with a mean of 17.1 years.
In total 56 AUFs were reported in 53 patients. The 6 AUFs in 6 patients diagnosed between 2003 and 2007 we presented earlier [2] were included in the incidence calculation, but not further described in this article. Of 14 cases, the exact details could not be retrieved. The details of the remaining 36 AUFs in 33 patients are presented in Tables 1 and 2.

Thus, within a timeframe of 16 years 56 AUFs were identified. On average 3.5 recognized AUFs/year. The Netherlands had 16.2 million inhabitants in 2003 and 17.5 million in 2021 [3].

\section{Demographics, diagnostics and AUF location (Table 1)}

The male to female ratio was 55\%:45\%. A history of oncological treatment and pelvic radiation was observed in 61 and 39\%, respectively, with a mean radiation dose of 64 Gray. The mean time between radiation and first presentation of AUF was 88 months [18-192 months]. The most frequent oncological diagnosis was bladder cancer in men (21\%) and cervical cancer in women (18\%). A medical history of (endo)vascular treatment was present in $33 \%$. Indwelling ureteral stents were present in 29 patients $(88 \%)$, reasons for placement were ureteral strictures due to radiation, retroperitoneal fibrosis or ureteral-ileal stenosis.

Hematuria was observed in all patients; $9 \%$ intermittent microhematuria (3/33 patients), and $91 \%$ (30/33 patients) building up to or initially presenting with massive hematuria. Accompanying symptoms were clot retention (21\%), flank pain (9\%), shock (6\%), sepsis (3\%), melena (3\%), and urosepsis (3\%).

All patients underwent a cumulative number of 40 investigations (mean 1.2 per patient) not leading to AUF diagnosis. For confirmation of the diagnosis of AUF, angiography was most decisive (19 times), followed by CT angiography (8 times), during surgery (4 times), ureterography (2 times), MRI (1 time), and postmortem (1 time).

Based on the details of clinical presentation, applied diagnostics and treatment, we presented a clinical algorithm, as presented in Supplementary Fig. 3.

Supplementary Fig. 4 shows the AUF locations. The common iliac artery was predominantly involved (73\%), right sided in $38 \%$ and left sided in $35 \%$. At diagnosis, seven patients with a medical history of vascular surgery on one side, such as thrombendarteriectomy or stenting, showed the AUF on the ipsilateral side of the repair and in 4 out of 7 patients a pseudoaneurysm was observed. In all six patients after central vascular repair, the AUF was found on the right side a pseudoaneurysm seen in three cases. 


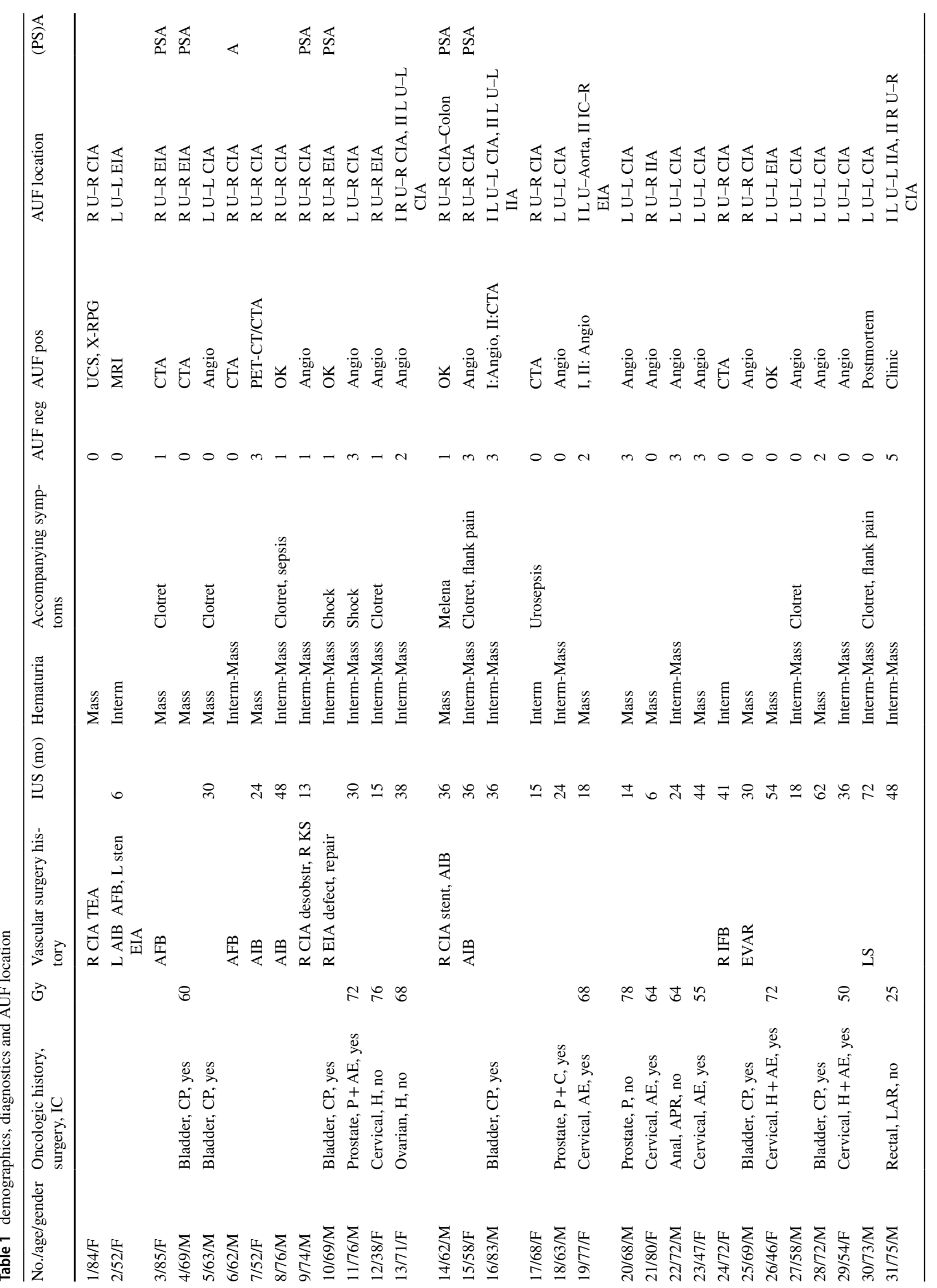




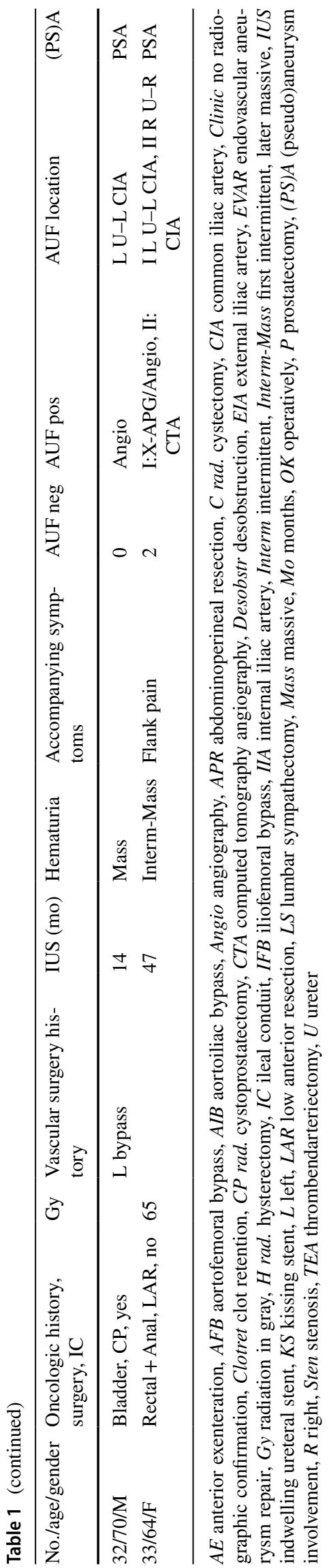

Treatment, follow-up, and outcomes (Table 2)

Open vascular repair was performed in 10/33 patients (30\%), urological treatment in $7 / 33$ patients $(21 \%)$, and endovascular stent graft placement in $24 / 33$ patients (73\%) with embolization of the internal iliac artery in eight patients.

A combined urological and (endo)vascular treatment was performed in $12 / 33$ patients $(36 \%)$.

In two patients nephroureterectomy was performed, as hematuria was considered to be of renal origin. Afterwards, AUF appeared to be the cause of the hematuria.

Follow-up ranged from 1.5 days to 92 months with a median of 35 months. Fifteen patients died during followup; two were AUF related, five were sepsis related, and eight were due to other causes.

The first AUF-related death was a patient with Chronic Obstructive Pulmonary Disease (COPD) Gold IV, who died 6 days after placement of an endovascular stent, due to sepsis and weaning difficulties on the ICU. The second AUF-related death was due to severe hemorrhage which was inadequately controlled. This patient died within 2 days after presentation and the definitive diagnosis was found at autopsy.

The five sepsis-related deceased patients died after 1-4 months (mean 3 months). Their deaths were possibly related to the initiation of infection after insertion of endovascular stent grafts $(3 \times)$ or vascular prosthesis during AUF treatment.

The causes of death not related to AUF were: metastatic disease in three patients after 11-34 months (mean 25 months), cardiovascular causes in two patients after, respectively, 35 and 50 months, a car accident in one patient after 84 months, probably COVID-19 in one patient after 41 months, and intestinal pneumatosis in one patient after 1 month.

This results in an overall mortality rate of $45 \%$ after a mean of 23 months, with a AUF-specific mortality rate of $6 \%$.

In four patients, a second AUF developed after 1 day and 4, 8, 12 months, respectively. All four patients were treated with an endovascular stent graft and survived with a followup of 92, 32, 50, and 15 months, respectively. One patient developed an arterio-conduit fistula (ACF) after 6 months, which was treated with an endovascular stent, and had an uneventful follow-up of 30 months. Besides these five patients with second AUF or ACF development, none of the other patients showed persistent hematuria. 
Table 2 treatment, outcome, follow-up (FU)

\begin{tabular}{|c|c|c|c|c|c|c|c|c|c|}
\hline$N$ & Unnec & Open treatment & Endovascular treatment & Emb IIA & Uro & FU & FU time & Reason death & Post-OK \\
\hline 1 & Nephr & & $\begin{array}{l}\text { WALLGRAFTTM } \\
10 \times 50+12 \times 50\end{array}$ & & & Surv & $48 \mathrm{~m}$ & & \\
\hline 2 & & Rem./IFB (GSV) & & & UU & Surv & $54 \mathrm{~m}$ & & \\
\hline 3 & & Femfem & & & UU & Died & $4 \mathrm{~m}$ & Sepsis & \\
\hline 4 & & Dacron $8 \times 50 \mathrm{~mm}$ & & & & Died & $4 \mathrm{~m}$ & Sepsis & \\
\hline 5 & & & $\begin{array}{l}\text { WALLGRAFTTM } \\
12 \times 50+\text { Zenith }^{\circledR} \\
14 \times 80\end{array}$ & & & Died & $84 \mathrm{~m}$ & Car accident & \\
\hline 6 & & & VIABAHN $^{\circledR} 13 \times 100$ & & & Surv & $21 \mathrm{~m}$ & & \\
\hline 7 & & & Advanta V1 $2^{\circledR} 10 \times 60$ & Yes & & Surv & $2 \mathrm{~m}$ & & \\
\hline 8 & & Lig. R CIA, Femfem & & & & Died & $35 \mathrm{~m}$ & Cardiovasc & Afunctional kidney \\
\hline 9 & & $\begin{array}{l}\text { Rem./Nitinol } 10 \times 79 \text {, } \\
\text { Advanta } 10 \times 59 \\
(\mathrm{KS})\end{array}$ & & & U-lysis & Surv & $28 \mathrm{~m}$ & & E Coli sepsis \\
\hline 10 & & & Endo $9 \times 38$ & Yes & & Died & $11 \mathrm{~m}$ & Cancer (bladder M+) & \\
\hline 11 & & & Jaguar $^{\mathrm{TM}} 45 \times 12$ & & & AUF & $6 \mathrm{~d}$ & Post-OK: pulm insuf & \\
\hline 12 & & & VIABAHN $^{\circledR} 60 \times 10$ & Yes & & Died & $3 \mathrm{~m}$ & Sepsis & IL FBV AE death \\
\hline 13 & & & $\begin{array}{l}\text { I VIABAHN }^{\circledR}, \text { II } \\
\text { VIABAHN }^{\circledR} 80 \times 10\end{array}$ & Yes & & Surv & $50 \mathrm{~m}$ & & $8 \mathrm{~m}:$ second AUF \\
\hline 14 & & $\begin{array}{l}\text { Rem./Dacron } \\
12 \times 50+V P\end{array}$ & & & & Surv & $36 \mathrm{~m}$ & & \\
\hline 15 & & & FLUENCY $^{\circledR} 12 \times 60$ & & & Died & $34 \mathrm{~m}$ & Cancer (lung) & \\
\hline 16 & & & $\begin{array}{l}\text { I FLUENCY }^{\circledR} 14 \times 40 \text {, } \\
\text { II Endo } 12 \times 40\end{array}$ & & & Surv & $15 \mathrm{~m}$ & & $12 \mathrm{~m}$ : second AUF \\
\hline 17 & & & Endo $9 \times 40$ & & & Died & $1 \mathrm{~m}$ & Pneumatosis intest & \\
\hline 18 & & & FLUENCY $^{\circledR} 12 \times 60$ & & & Died & $50 \mathrm{~m}$ & Cardiovasc & \\
\hline 19 & & I VP, omental wrap & II FLUENCY ${ }^{\circledR} 10 \times 60$ & Yes & & Surv & $36 \mathrm{~m}$ & & $6 \mathrm{~m}: \mathrm{ACF}$ \\
\hline 20 & & & VIABAHN $^{\circledR} 12 \times 60$ & & Balloon & Surv & $72 \mathrm{~m}$ & & \\
\hline 21 & & Lig. R IIA & Advanta V12 $2^{\circledR} 8 \times 59$ & & URI & Died & $2 \mathrm{~m}$ & Sepsis, cardiovasc & \\
\hline 22 & & & VIABAHN $^{\circledR} 8 \times 50$ & & & Surv & $60 \mathrm{~m}$ & & \\
\hline 23 & & & FLUENCY $^{\circledR} 12 \times 60$ & & & Surv & $60 \mathrm{~m}$ & & \\
\hline 24 & & Rem./IFB (GSV) & & & & Surv & $59 \mathrm{~m}$ & & \\
\hline 25 & & & VIABAHN $^{\circledR}$ & Yes & & Died & $1 \mathrm{~m}$ & Sepsis, cardiovasc & \\
\hline 26 & & IFB & & & URI & Surv & $61 \mathrm{~m}$ & & \\
\hline 27 & Nephr & & EVAR limb & & & Surv & $72 \mathrm{~m}$ & & \\
\hline 28 & & & FLUENCY $^{\circledR} 10 \times 50$ & Yes & & Surv & $60 \mathrm{~m}$ & & \\
\hline 29 & & & VIABAHN $^{\circledR} 10 \times 50$ & Yes & & Surv & $18 \mathrm{~m}$ & & \\
\hline 30 & & & & & & AUF & $1.5 \mathrm{~d}$ & Mass hemorrhage & \\
\hline 31 & & & $\begin{array}{l}\text { II GORE }^{\circledR} \\
\quad \text { EXCLUDER }^{\circledR} 15 \times 70\end{array}$ & I Yes & & Surv & $92 \mathrm{~m}$ & & $<1 \mathrm{~d}$ : second AUF \\
\hline 32 & & & BeGraft $^{\circledR} 10 \times 38$ & & & Died & $41 \mathrm{~m}$ & Possible COVID & $30 \mathrm{~m}$ : infection graft \\
\hline 33 & & & $\begin{array}{l}\text { I FLUENCY }^{\circledR} 10 \times 60, \\
\text { II FLUENCY } \\
\text { II }^{\circledR} 8 \times 40\end{array}$ & Yes & Allium & Died & $32 \mathrm{~m}$ & Cancer (Anal M+) & $\begin{array}{l}\text { Rec.: } 2 \text { extra endo } \\
\text { stents }\end{array}$ \\
\hline
\end{tabular}

$A C F$ arterial-conduit fistula, $A E$ anterior exenteration, $A U F$ AUF-related death, Balloon temporary balloon tamponade, cardiovasc cardiovascular, CIA common iliac artery, $d$ day, emb embolization, endo endovascular stent (name not known), $F B V$ fistula bladder-vagina, femfem femoralfemoral bypass, $G S V$ great saphenous vein, IFB iliofemoral bypass, $I L$ intestinal leak, intest intestinalis, $K S$ kissing stent, lig ligation, $m$ months, mass massive, $n$ case number, nephr nephrectomy, pulm insuff pulmonary insufficiency, rec recurrent hematuria, rem./ removal of old stents, surv survived, $u$-lysis ureterolysis, unnec unnecessary treatment, $U R I$ ureteral reimplantation, $U U$ ureteroureterostomy, $V P$ venous patch, $x$ diameter $\times$ length $(\mathrm{mm})$ stent 


\section{Discussion}

This nationwide cross-sectional questionnaire analysis comprehensively presents the occurrence of AUF in The Netherlands. Based on the results, risk factors, location, and clinical presentation of AUF was described. We also presented an overview of performed diagnostic modalities, and treatment of AUF in a modern healthcare system as in The Netherlands.

Our main finding is the incidence of AUF in The Netherlands per year. The calculated incidence in The Netherlands of AUF was 3.5 AUFs/year. The true incidence is likely to be higher, considering the non-response by $38 \%$ of urologists. In case the incidence of AUF in the practices of non-responders was comparable to those of the responders, incidence of AUF would actually be 5.6 per year (90/16). Calculations have to be interpreted with caution. The questionnaire was only sent to urologists. Some cases of AUF where urologists were not involved might have been missed. In addition patients may have died because of AUF in whom the diagnosis has not been made at all. Finally, recall bias may have played a roll.

In the literature, no figures of incidence changes over the past few years can be found. However, larger series have been published in recent years.

Krambeck et al. [4] only describe seven AUF patients between 1975 and 2004, while Omran et al. [5] and Simon et al. [6] describe 25 AUF patients between 2011 and 2020 and 16 AUF patients between 2005 and 2020, respectively. This suggests that the reported incidence is increasing.

This increase may be due to more extensive pelvic treatments for varying oncological and vascular procedures in current surgical medicine, increased treatment with ureteral indwelling stents, more frequent radiation therapies, and overall longer life expectancy. Other possible reasons are improving imaging modalities, and improvement of medical registration and electronic patient files, leading to easier identification of AUF cases. It is difficult to estimate the true incidence based on the numbers of published cases, because publication bias cannot be ruled out.

All our patients had a history of oncological treatment, irradiation therapy, vascular pelvic surgery, or chronic ureteral indwelling stents. Thus, all our patients had fistulas secondary to other conditions. This is in accordance with the findings described in Bergqvist and Madoff $[7,8]$, who found that $85 \%$ of the fistulas were secondary fistulas. Primary fistulas are probably rare or may remain unrecognized.

Although the pathophysiology of AUF is still uncertain, different hypotheses exist on the development of AUF, and the role of risk factors (Fig. 1). Surgery and radiation therapy lead to ischemia and fibrosis with fixation of a frail ureter to an artery (Fig. 1b). Ureteral indwelling stents can increase the damage due to pressure, facilitating necrosis and, eventually, fistula formation (Fig. 1c) $[8,9]$.

The male to female ratio was 55\%:45\%. This is in contrast with most cohort studies, such as Subiela et al. [10], who found a female predilection (59.6\%). An explanation could be that bladder cancer was the predominant type of cancer and this is more common in the male population.

The clinical presentation in the majority of our patients was long-lasting intermittent (micro)hematuria that promptly shifted to massive hemorrhage, especially during ureteral stent extraction or replacement; only three cases (9\%) had intermittent microhematuria without clinical consequences as the only symptom. This clinical presentation mirrors that of previous studies [11].
Fig. 1 Illustration to explain the pathophysiology of AUF. A Normal condition: a freely movable ureter with a pulsatile artery. The artery not affecting the ureter. B Pelvic surgery and/or radiation could cause fibrosis and ischemic injury. This leads to ureter obstruction and hydronephrosis and fixation of the ureter to the arterial wall. C Ureteral stent placement to treat hydronephrosis which causes friction due to less freely movable ureter. In time, fibrosis, ischemia and/or friction could cause localized necrosis and eventually AUF
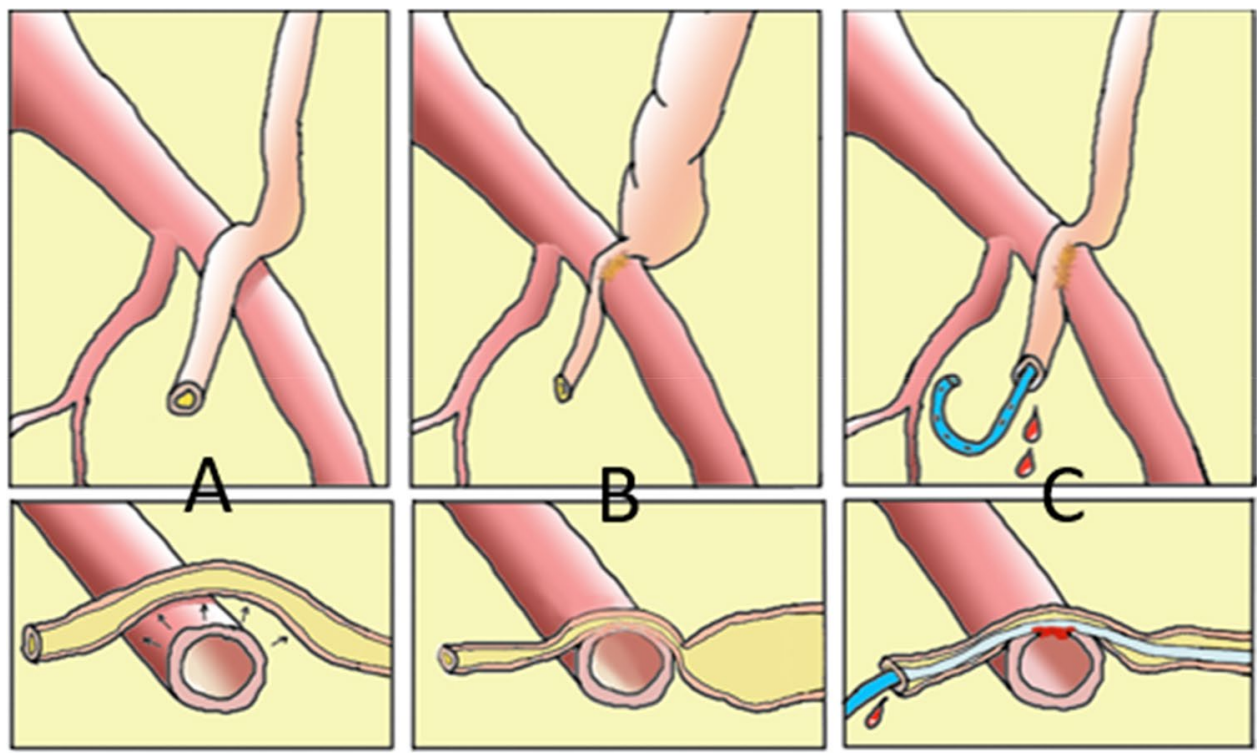
Several abdominal imaging modalities failed to confirm the diagnosis AUF. In our patient population cohort 38 investigations/imaging procedures were performed which not lead to the correct diagnosis. In two CT angiographies renal clots suggested a renal bleeding, leading to unnecessary nephroureterectomy. Angiography seems to be the most efficient modality, confirming diagnosis in 19/33 patients (58\%). However, a negative angiography could not exclude AUF, because active bleeding is necessary for reliable angiography [12]. In case of negative angiography, provocative angiography (ureteral or vascular manipulation at AUF site) should be considered under controlled circumstances [12]. Diagnosis of AUF remains challenging because of low sensitivity of imaging tests.

Therapeutic management in The Netherlands is predominantly endovascular (73\%) with the placement of stent grafts during angiography. In this century there has been a shift in the therapeutic management of AUF from a surgical toward a less invasive approach [9]. In the last decade, combination of therapies are more frequently performed. In the acute phase vascular and urologic surgeons work together in open surgery or the interventional radiologist starts with placing an endovascular stent graft and the vascular surgeon follows with a delayed open reconstruction (extra-anatomic bypass) or treating of graft infection and stent thrombosis [5, 13]. One of the measures to prevent future fistula formation may include considering nephrostomy instead of chronic indwelling stent in patients with multiple risk factors. A multidisciplinary approach is important for adequate diagnosis and treatment.

The AUF-specific mortality rate in our cohort was $6 \%$, which is at the lower end of the spectrum. Five patients had sepsis-related death likely due to the endovascular stent grafts placed during AUF-treatment. However, this occurred after more than 30 days, so was not calculated in the AUFspecific mortality.

In previous studies the reported AUF-related mortality was rather variable: Escobar et al. found 10-13\% and 22\% in the hemodynamic instable patients [14], van den Bergh et al. described 13\% AUF-related mortality [1], and more recently published Heers et al. calculated an AUF-related mortality of $7.7 \%$ [9].

The overall mortality was $45 \%$ after a median of 4 months. A possible explanation for this high rate is limited overall prognosis due to oncological and/or vascular comorbidity [15].

Noteworthy, in our cohort four new secondary AUFs were seen within 1 year and one ACF, all successfully treated by an endovascular stent. Simon et al. [6] and Omran et al. [5] describe recurrent AUFs, but no literature was found on new secondary AUFs. A possible explanation could be that these patients were $>70$ years and all had radiation therapy in the past.

\section{Strengths and limitations}

To our knowledge this is the first nationwide cross-sectional questionnaire analysis for AUF and the largest series of new AUF patients. One of the main strengths is that the response rate of $62 \%$ is quite high compared to overall response rate on similar questionnaires. This adequately reflects all urologists in The Netherlands.

Our study is the first to address the occurrence of AUF and contemporary experience by urologists on a national level. However, our study is limited by recall bias, possible missed diagnosis, and the retrospective nature.

\section{Conclusions}

The estimated incidence of AUF in the time period 2003-2018 in The Netherlands is close to 4 AUFs per year, but the true rates may be higher. In 56 cases described in this series, hematuria, sometimes massive, was the most predominant initial symptom. Angiography appears the best diagnostic modality. Treatment was predominantly endovascular intervention.

We identified previous oncological and vascular treatment as well as indwelling ureteral catheters as major risk factors. Reported mortality rate was $6 \%$ and may be underestimated due to missed diagnoses.

AUF should be considered in patients with persistent intermittent or massive hematuria, especially in combination with history of oncologic (radiation) therapy, as well as vascular surgery.

Clinical awareness of AUF is important because of its possibly life threatening nature. A multidisciplinary approach is important in diagnosis and treatment.

Supplementary Information The online version contains supplementary material available at https://doi.org/10.1007/s00345-021-03910-3.

Author contributions MTWTL: Protocol/project development, Data collection or management, Data analysis, Manuscript writing/editing. KK: Protocol/project development, Data collection or management, Data analysis, Manuscript writing/editing. RCNB: Manuscript writing/ editing. FLM: Manuscript editing. JPPMV: Manuscript editing. THL: Manuscript editing. GAPK: Manuscript editing. RCGB: Manuscript editing. PD: Manuscript editing, drawings. SH: Manuscript editing, supervisor. LMOK: Manuscript editing, supervisor.

\section{Declarations}

Conflict of interest No conflict of interest.

Research involving human participants and/or animals The Ethics Committee of the University Medical Center Utrecht approved this study (reference number WAG/mb/19/042283). The Dutch Urological Association approved questionnaire distribution among urologists. 
Informed consent The need for individual consent was waived.

Open Access This article is licensed under a Creative Commons Attribution 4.0 International License, which permits use, sharing, adaptation, distribution and reproduction in any medium or format, as long as you give appropriate credit to the original author(s) and the source, provide a link to the Creative Commons licence, and indicate if changes were made. The images or other third party material in this article are included in the article's Creative Commons licence, unless indicated otherwise in a credit line to the material. If material is not included in the article's Creative Commons licence and your intended use is not permitted by statutory regulation or exceeds the permitted use, you will need to obtain permission directly from the copyright holder. To view a copy of this licence, visit http://creativecommons.org/licenses/by/4.0/.

\section{References}

1. van den Bergh RCN, Moll FL, de Vries J-PPM, Lock TMTW (2009) Arterioureteral fistulas: unusual suspects-systematic review of 139 cases. Urology 74(2):251-255. https://doi.org/10. 1016/j.urology.2008.12.011

2. Van den Bergh RCN, Moll FL, De Vries J-PPM, Yeung KK, Lock TMTW (2008) Arterio-ureteral fistula: 11 new cases of a wolf in sheep's clothing. J Urol 179(2):578-581. https://doi.org/10.1016/j. juro.2007.09.087

3. CBS: population counter The Netherlands. https://www.cbs.nl/ en-gb/visualisations/dashboard-population/population-counter

4. Krambeck AE, DiMarco DS, Gettman MT, Segura JW (2005) Ureteroiliac artery fistula: diagnosis and treatment algorithm. Urology 66(5):990-994. https://doi.org/10.1016/j.urology.2005. 05.036

5. Omran S, Schäfer H, Kapahnke S et al (2020) Endovascular and open surgical options in the treatment of uretero-arterial fistulas. Vascular. https://doi.org/10.1177/1708538120970823

6. Simon B, Neubauer J, Schoenthaler M, Hein S, Bamberg F, Maruschke L (2021) Management and endovascular therapy of ureteroarterial fistulas: experience from a single center and review of the literature. CVIR Endovasc 4(1):36. https://doi.org/10.1186/ s42155-021-00226-6

\section{Authors and Affiliations}

Tycho M. T. W. Lock ${ }^{1}$ (D) Kyara Kamphorst ${ }^{1} \cdot$ Roderick C. N. van den Bergh $^{2} \cdot$ Frans L. Moll $^{3} \cdot$ Jean-Paul P. M. de Vries $^{4}$. Rob T. H. Lo ${ }^{5}$. Gérard A. P. de Kort ${ }^{5}$ - Rutger C. G. Bruijnen ${ }^{5}$ - Pieter Dik ${ }^{6,7} \cdot$ Simon Horenblas $^{8} \cdot$ Laetitia M. O. de Kort $^{1}$

Kyara Kamphorst

k.kamphorst2@students.uu.nl

Roderick C. N. van den Bergh

r.van.den.bergh@antoniusziekenhuis.nl

Frans L. Moll

fransmol150@gmail.com

Jean-Paul P. M. de Vries

j.p.p.m.de.vries@umcg.nl

Rob T. H. Lo

t.h.lo@umcutrecht.nl

Gérard A. P. de Kort

G.A.P.deKort@umcutrecht.nl

Rutger C. G. Bruijnen

R.Bruijnen@umcutrecht.nl
7. Bergqvist D, Pärsson H, Sherif A (2001) Arterio-ureteral fistulaa systematic review. Eur J Vasc Endovasc Surg 22(3):191-196. https://doi.org/10.1053/ejvs.2001.1432

8. Madoff DC, Gupta S, Toombs BD et al (2004) Arterioureteral fistulas: a clinical, diagnostic, and therapeutic dilemma. AJR Am J Roentgenol 182(5):1241-1250. https://doi.org/10.2214/ajr.182.5. 1821241

9. Heers H, Netsch C, Wilhelm K et al (2018) Diagnosis, treatment, and outcome of arterioureteral fistula: the urologist's perspective. J Endourol 32(3):245-251. https://doi.org/10.1089/end.2017.0819

10. Subiela JD, Balla A, Bollo J et al (2018) Endovascular management of ureteroarterial fistula: single institution experience and systematic literature review. Vasc Endovasc Surg 52(4):275-286. https://doi.org/10.1177/1538574418761721

11. Matsunaga F, Dambaeva A, Ponsky LE, Kashyap VS, Tavri S (2021) Systematic review of the management of ureteroarterial fistulas after ileal conduit urinary diversion. AJR Am J Roentgenol. https://doi.org/10.2214/AJR.20.23132

12. Augustin AM, Torre GD, Kocot A, Bley TA, Kalogirou C, Kickuth R (2021) Endovascular therapy of arterioureteral fistulas. Vasa 50(3): 193-201. https://doi.org/10.1024/0301-1526/a000922

13. Titomihelakis G, Feghali A, Nguyen T, Salvatore D, DiMuzio P, Abai B (2019) Endovascular management and the risk of late failure in the treatment of ureteroarterial fistulas. J Vasc Surg cases Innov Tech 5(4):396-401. https://doi.org/10.1016/j.jvscit.2019. 06.010

14. Escobar PF, Howard JL, Kelly J et al (2008) Ureteroarterial fistulas after radical pelvic surgery: pathogenesis, diagnosis, and therapeutic modalities. Int J Gynecol Cancer Off J Int Gynecol Cancer Soc 18(4):862-867. https://doi.org/10.1111/j.1525-1438. 2007.01079.x

15. Noordzij PG, Poldermans D, Schouten O, Bax JJ, Schreiner FAG, Boersma E (2010) Postoperative mortality in The Netherlands: a population-based analysis of surgery-specific risk in adults. Anesthesiology 112(5):1105-1115. https://doi.org/10.1097/ALN. 0b013e3181d5f95c

Publisher's Note Springer Nature remains neutral with regard to jurisdictional claims in published maps and institutional affiliations.
Pieter Dik

pieter.dik@gmail.com

Simon Horenblas

s.horenblas@nki.nl

Laetitia M. O. de Kort

1.m.o.dekort@umcutrecht.nl

1 Department of Urology, University Medical Centre Utrecht, P.O. BOX 85500, 3508 GA Utrecht, The Netherlands

2 Department of Urology, St. Antonius Hospital, Koekoekslaan 1, 3435 CM Nieuwegein, The Netherlands

3 Division of Vascular Surgery, Department of Surgery, University Medical Centre Utrecht, P.O. BOX 85500, 3508 GA Utrecht, The Netherlands 
4 Division of Vascular Surgery, Department of Surgery, University Medical Centre Groningen, Hanzeplein 1, 9713 GZ Groningen, The Netherlands

5 Department of Interventional Radiology, University Medical Centre Utrecht, P.O. BOX 85500, 3508 GA Utrecht, The Netherlands

6 Department of Pediatric Urology, Wilhelmina Children's Hospital UMC Utrecht, Utrecht, The Netherlands
7 Division of Pediatric Urology and Andrology, Department of Pediatric Surgery, I.M. Sechenov First Moscow State Medical University, Moscow, Russia

8 Department of Urology, The Netherlands Cancer Institute, Plesmanlaan 121, 1066 CX Amsterdam, The Netherlands 